\section{Cerebral Air Embolism: A Result of Atrioesophageal Fistula}

Cerebral air embolism is a rare but known complication resulting from intracardiac instrumentation. Although reports of atrioesophageal (AE) fistulas have been documented in clinical journals ${ }^{1}$ and the cardiothoracic radiology literature, ${ }^{2}$ there has been little focus on the neurologic manifestations and imaging findings of this entity. We report the case of an air embolism presenting 35 days after surgery for refractory atrial fibrillation.

A 63-year-old man presented to our hospital with fever, chills, malaise, and left arm weakness. Five weeks before presentation he had undergone a partial Cox-Maze catheter-directed cryoablation as a treatment for refractory atrial fibrillation. Blood cultures drawn on admission had grown various streptococcal and staphylococcal species. After the patient developed altered mentation, an MR imaging of the brain was performed and demonstrated multiple punctate foci of restricted diffusion predominantly in the right cerebral hemisphere (Fig $1 A,-B$ ). A follow-up head CT showed curvilinear areas of very low attenuation $(-600 \mathrm{HU})$ within several cerebral sulci, suggesting air emboli (Fig 2). With a recent history of cardiothoracic intervention and an unknown source of bacteremia, a chest CT scan was obtained searching for a source of septic emboli or air emboli. Chest CT findings included a sizeable focus of air in the left atrium and a smaller amount of extracardiac air along the anterior wall of the esophagus (Fig 3). The patient underwent emergent exploratory and corrective surgery, at which time an AE fistula was confirmed. The postoperative course was uneventful, and the patient is currently recovering.

Cerebral air embolism is often an iatrogenic complication of an invasive procedure. ${ }^{3} \mathrm{AE}$ fistulas are a known complication of intraoperative cardiac ablations with an incidence reported to be $1 \%{ }^{2}$ along with a high rate of mortality. However, most air emboli are caused by introduction of gas into the venous system as a result of trauma, central line placement, or cardiothoracic or neurologic surgery. The mechanism by which air then enters the systemic circulation may relate to an anatomic cardiac defect, or in certain conditions, it may involve oxygen toxicity and excessive volumes of gas, either of which can lead to passage of air bubbles through the pulmonary vasculature.

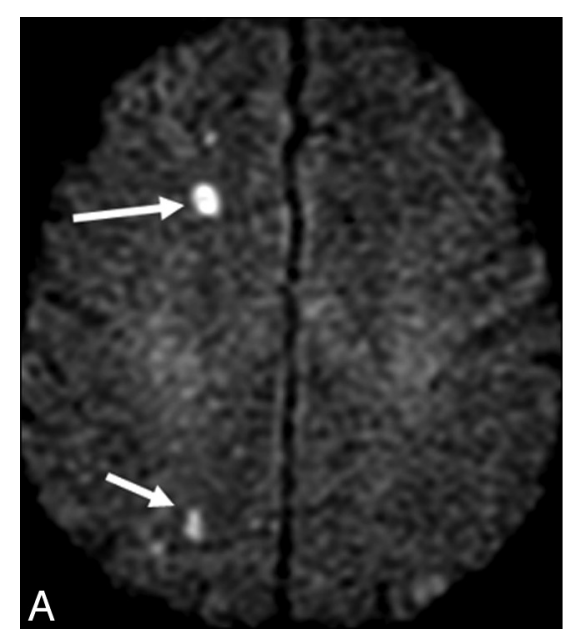

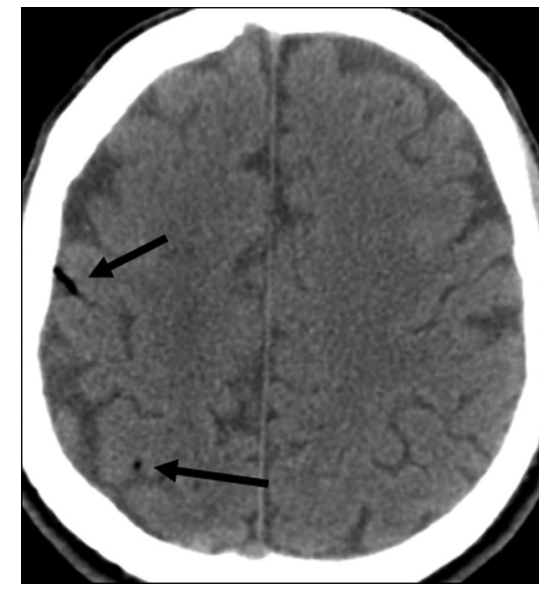

Fig 2. Noncontrast head CT scan shows several foci of air within cortical vessels, suggesting an air embolism (arrows).

Our case demonstrates a rare situation in which air was introduced directly into the systemic circulation via an iatrogenic AE fistula. The classic course of arterial air emboli is to enter the first major branch off the aorta, the innominate artery, and to proceed to the right carotid circulation. ${ }^{3}$ Most infarcts occurred in the right cerebral hemisphere in our case, exemplifying this anatomic pathway. The presumed pathophysiology of the MR imaging findings involved cerebral edema due to capillary leak syndrome and ischemia from arterial occlusion.

Current treatments attempt to eliminate intravascular air and provide cardiac massage and supplementary oxygenation. Hyperbaric oxygen, which diminishes gas volume and cerebral edema and enhances partial pressure of dissolved oxygen in the blood, is indicated in the presence of neurologic deficits. ${ }^{1}$ However, it is imperative that the radiologist recognize an air embolus and recommend early intervention because the patient will continue to decline if the underlying pathology is not repaired.

\section{References}

1. Blanc $\mathrm{P}$, Boussuges A, Henriette $\mathrm{K}$, et al. Iatrogenic cerebral air embolism: importance of an early hyperbaric oxygenation. Intensive Care Med 2002;28:559-63

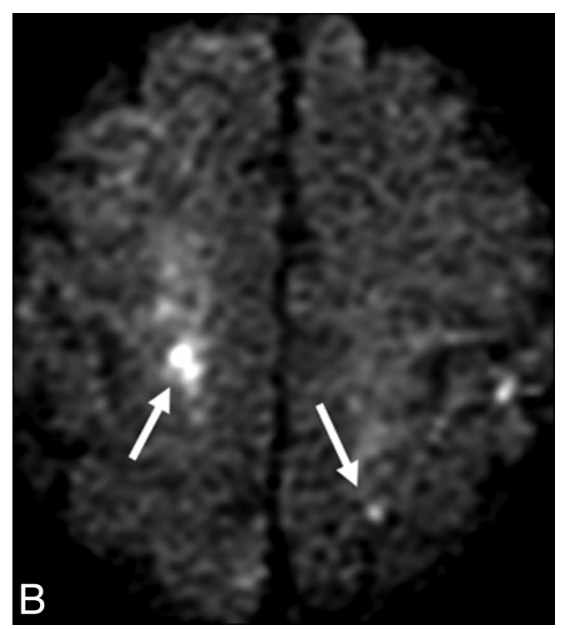

Fig 1. Brain MR images with DWI demonstrate multiple punctate foci of restricted diffusion (arrows) predominantly in the right cerebral hemisphere, indicating infarcts resulting from cerebral air emboli. 


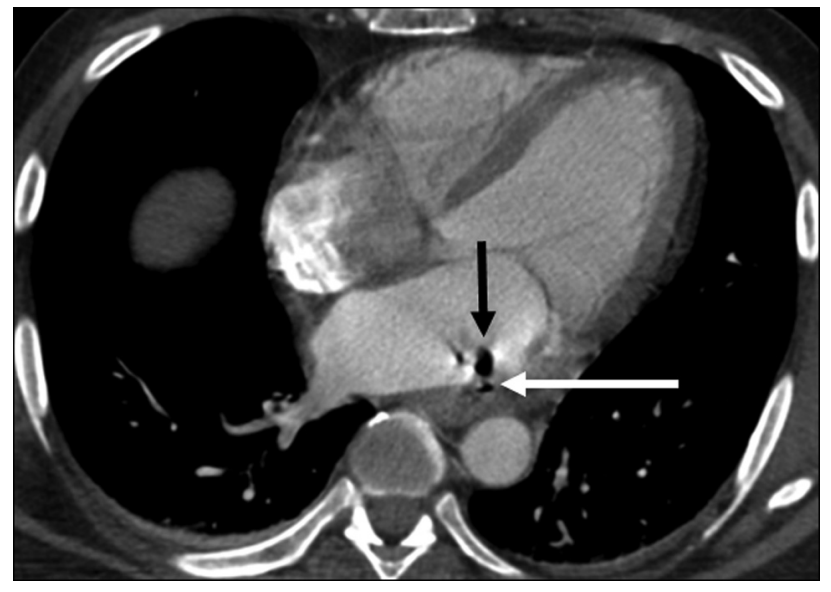

Fig 3. Contrast-enhanced chest CT scan shows a bubble of air in the left atrium (black arrow) and a smaller amount of extracardiac air along the anterior wall of the esophagus (white arrow), consistent with an AE fistula.
2. Preis O, Digumarthy SR, Wright CD, et al. Atrioesophageal fistula after catheter pulmonary venous ablation for atrial fibrillation: imaging features. J Thorac Imaging 2007;22:283-85

3. Jensen ME, Lipper MH. CT in iatrogenic cerebral air embolism. AJNR Am J Neuroradiol 1986;7:823-27

J.K. Lempel

Department of Radiology

B. Jozwik

Department of Medicine

C. Manfredi

Department of Medicine

A.C. Shih

Department of Radiology

Norwalk Hospital

Norwalk, Connecticut 